\title{
A Longitudinal Study of Structural Risk Factors for Obesity and Diabetes Among American Indian Young Adults, 1994-2008
}

\author{
Tennille L. Marley, PhD, MPH; Molly W. Metzger, PhD
}

\begin{abstract}
Suggested citation for this article: Marley TL, Metzger MW. A Longitudinal Study of Structural Risk Factors for Obesity and Diabetes Among American Indian Young Adults, 1994-2008. Prev Chronic Dis 2015;12:140469. DOI: http://dx.doi.org/ $10.5888 / \mathrm{pcd} 12.140469$.
\end{abstract}

\section{PEER REVIEWED}

\section{Abstract}

\section{Introduction}

American Indian young adults have higher rates of obesity and type 2 diabetes than the general US population. They are also more likely than the general population to have higher rates of structural risk factors for obesity and diabetes, such as poverty, frequent changes of residence, and stress. The objective of this study was to investigate possible links between these 2 sets of problems.

\section{Methods}

Data from the American Indian subsample of the National Longitudinal Study of Adolescent to Adult Health (Add Health) were used to examine potential links between obesity and type 2 diabetes and structural risk factors such as neighborhood poverty, housing mobility, and stress. We used logistic regression to explore explanatory factors.

\section{Results}

American Indians in the subsample had higher rates of poor health, such as elevated hemoglobin A1c levels, self-reported high blood glucose, self-reported diabetes, and overweight or obesity. They also had higher rates of structural risk factors than non-Hispanic whites, such as residing in poorer and more transient neighborhoods and having greater levels of stress. Self-reported stress partially mediated the increased likelihood of high blood glucose or diabetes among American Indians, whereas neighborhood poverty partially mediated their increased likelihood of obesity.

\begin{abstract}
Conclusion
Neighborhood poverty and stress may partially explain the higher rates of overweight, obesity, and type 2 diabetes among American Indian young adults than among non-Hispanic white young adults. Future research should explore additional neighborhood factors such as access to grocery stores selling healthy foods, proximity and safety of playgrounds or other recreational space, and adequate housing.
\end{abstract}

\section{Introduction}

Rates of overweight, obesity, and type 2 diabetes are growing in the United States across all racial and ethnic groups and among children and adolescents (1-4). However, American Indian adolescents and young adults are more likely than adolescents and young adults of other races and ethnicities to have these conditions $(4,5)$. American Indian adolescents are more likely to be overweight or obese (42\%) than non-Hispanic whites (26.7\%), Latinos (37.6\%), and African Americans (41.1\%) (2). From 1990 to 1998, type 2 diabetes diagnoses increased by $71 \%$ among American Indian children, adolescents, and young adults and prevalence increased by $68 \%$ (from 1.23 per 1,000 to 5.42 per 1,000 ) among American Indian adolescents aged 15 to 19 (5). Overweight and obesity can have serious consequences for health, including cardiovascular disease, type 2 diabetes, and other conditions that can contribute to lower quality of life, disability, and premature death (6).

Structural determinants and conditions of daily life make up the social determinants of health and are responsible for many poor health outcomes, and increasingly, researchers recognize the effects of various social determinants that contribute to overall health (7-9). A growing body of research suggests that disease and ill health are largely the result of the "circumstances in which people are born, grow, live, work, and age, and the systems put in place to deal with illness" (9). Despite evidence of the associations among social determinants and health, empirical research on possible links between neighborhood factors and obesity, overweight, and type 2 diabetes among American Indians is scarce 
$(3,10-13)$. The objective of this study was to explore the associations between the structural determinants of neighborhood factors, parent education and obesity, and perceived stress with overweight, obesity, and type 2 diabetes among American Indian young adults.

\section{Methods}

\section{Survey design}

This study used data from the first and fourth waves of the National Longitudinal Study of Adolescent to Adult Health (Add Health), a nationally representative study following adolescents into early adulthood (14). These waves were chosen to capitalize on the rich neighborhood data available in Wave 1 and the multiple outcomes related to type 2 diabetes and obesity in Wave 4.

Wave 1 comprised adolescents in grades 7 through 12 (ages 12-19) in school year 1994-1995. Participants originated from a stratified random sample of 20,745 adolescents attending 80 high schools and 52 middle schools. The schools were stratified into 80 clusters, by variables such as region (Northeast, Midwest, South, West), urbanicity (urban, suburban, rural), school type (public, private, parochial), and other characteristics. In addition to the surveys of the adolescents themselves, 17,670 parents also completed interviews at Wave 1 . Wave 4 followed up with those adolescents when they were young adults aged 24 to 32 in 2007-2008. The Wave 4 follow-up included $76 \%$ of the original sample $(\mathrm{n}=15,701)$.

Attrition between Waves 1 and 4 differed by race and ethnicity. The Wave 4 response rate was highest for white participants (79\%) and lowest for Asian participants (66\%). The response rate was slightly below average for American Indian participants (73\%). To adjust for this differential response, all analyses used Add Health's longitudinal sampling weights designed for the Wave 4 sample ("pweights" in Stata [StataCorp LP]). These weights adjust for complex sample design, selection, and nonresponse, including adjustment for differential response by race, education level, and marital status (15). Overall, complete data were available for 12,657 respondents.

\section{Measures}

\section{Dependent variables}

Analyses included 4 outcomes: glycated hemoglobin (HbAlc), self-reported high blood glucose or type 2 diabetes, overweight/ obesity, and obesity. For HbA1c, whole-blood spot assays were collected via finger pricks, and levels were determined from colorimetric methods. HbA1c values greater than 5.7 were considered elevated.
In addition to the direct measure of $\mathrm{HbAlc}$, we examined self-reports of high blood glucose or type 2 diabetes, measured with a single item, "Has a doctor, nurse, or other health care provider ever told you that you have or had high blood sugar or diabetes?" We used direct measures of height and weight to calculate body mass index (BMI, $\mathrm{kg} / \mathrm{m}^{2}$ ). Overweight/obesity was defined as a BMI greater than or equal to 25.0 and obesity as a BMI greater than or equal to 30.0 .

\section{Race and ethnicity}

Racial and ethnic classifications were based on self-report at Wave 1. Our primary group of interest, American Indians, included participants who selected "Native American" solely or in combination with another racial or ethnic group. The Hispanic category included those self-reporting as Hispanic solely or in combination with another group (not including Native American). The white, black, and Asian categories comprised those self-reporting as each of those groups not in combination with another group. Because of small sample sizes, participants self-reporting as other combinations of racial and ethnic groups were classified as "other."

\section{Context measures}

Three measures of neighborhood characteristics were included in the analyses: neighborhood collective efficacy (ie, social cohesion), neighborhood poverty rate, and neighborhood mobility. Neighborhood collective efficacy was reported by adolescents in Wave 1 (16) and was calculated as the sum of 3 dichotomous (true/false) items: "You know most of the people in your neighborhood," "In the past month, you have stopped on the street to talk with someone who lives in your neighborhood," and "People in this neighborhood look out for each other." For each item, a no response was scored as zero, and a yes response was scored as 1 . Data for the second and third neighborhood measures were from 1990 Census block group data. Neighborhood poverty rate is the percentage of people living below the official poverty threshold (\$13,254 for a family of 4 in 1990). "Neighborhood mobility" was measured as the percentage of occupied housing units into which people moved during the previous 5 years. In addition to the neighborhood measures, dummy variables were included to reflect participants' school location in suburban, rural, or urban locations at Wave 1.

\section{Additional variables}

A robust set of control variables and mediators was included. Control variables from Wave 1 were adolescent-reported age, sex, and parent's highest level of educational attainment (for 2-parent families, data were used for the parent with the higher level of education). From Wave 4, we included the Cohen Perceived Stress Scale (PSS) $(17,18)$. The PSS score was calculated as the sum of 4 items (range, 0-16). Participants reported how often during the

\footnotetext{
The opinions expressed by authors contributing to this journal do not necessarily reflect the opinions of the U.S. Department of Health and Human Services, the Public Health Service, the Centers for Disease Control and Prevention, or the authors' affiliated institutions.
} 
previous 30 days they 1) were unable to control important things in their lives, 2) felt confident in their ability to handle their personal problems (reversed), 3) felt things were going their way (reversed), and 4) felt that difficulties were piling up so high that they were unable to overcome them. Each item was scored as 0 (never), 1 (almost never), 2 (sometimes), 3 (fairly often), or 4 (very often).

\section{Statistical analysis}

We calculated descriptive statistics (percentages, means, and 95\% confidence intervals) for the full sample and American Indian subsample. We calculated adjusted odds ratios (AORs) and $P$ values (significance set at an $\alpha$ level of .05) from a series of logistic regression models predicting elevated HbA1c, self-reported high blood glucose, and self-reported diabetes. Logistic regression models, neighborhood predictors, and perceived stress were entered as $Z$ scores for ease of comparison across coefficients. Finally, Sobel tests were conducted as a test of mediation. All analyses were implemented in Stata version 12 (StataCorp LP). Procedures for data access and analysis were implemented as approved by the institutional review board at Northwestern University and in agreement with the sensitive data security plan approved by Add Health data managers.

\section{Results}

Our analytic sample comprised 11,110 participants, including 393 participants who self-identified as American Indian (Table 1). At Wave 1 , the full sample resided in neighborhoods with a poverty rate of $13.9 \%$, whereas the American Indian subsample resided in neighborhoods with an average neighborhood poverty rate of 19.2\%. Neighborhood mobility was higher for the American Indian subsample than for the full sample; $49.3 \%$ in the subsample and $46.5 \%$ in the full sample of neighbors resided in the neighborhood for less than 5 years. The mean score for neighborhood collective efficacy was 0.75 for both the American Indian subsample and the full sample. At Wave 4, the mean score on the Cohen PSS was higher among American Indians (score, 5.6) than among the full sample (score, 4.8.)

The American Indian subsample was more likely than the full sample to have health problems across multiple indicators at Wave 4: $43.8 \%$ of the American Indian subsample had elevated HbA1c levels, compared with $30.6 \%$ of the full sample; $5.2 \%$ of the American Indian subsample reported having been told they had high blood glucose or type 2 diabetes, compared with $2.6 \%$ of the full sample; $76.8 \%$ of the American Indian subsample was overweight/obese or obese, compared with $66.6 \%$ of the full sample; and $42.5 \%$ of the American Indian sample was obese, compared with $37.4 \%$ of the full sample.
All racial/ethnic minority groups included in our logistic regression models were more likely than non-Hispanic whites to have elevated HbA1c (Table 2). In Model 1 (no control variables), American Indians were 2.66 times as likely as non-Hispanic whites to have elevated HbA1c $(P<.01)$; in Model 2 (controls for sex, age, parent education, and parent obesity), they were 2.47 times as likely $(P<.01)$. In Model 3 (further addition of controls for neighborhood variables, urbanicity, and perceived stress), the adjusted odds ratio (AOR) of American Indians having elevated HbA1c was further attenuated to 2.41 ; one of the 3 neighborhood variables (neighborhood collective efficacy) was significantly associated with elevated $\mathrm{HbA1c}$ (AOR, 1.07; $P=.04$ ); perceived stress was not. In Model 4 (addition of overweight/obesity and obesity), both overweight/obesity and obesity predicted elevated HbA1c ( $P$ $<.01$ for both). In this model, the likelihood of elevated HbA1c among American Indians was attenuated with the inclusion of overweight/obesity and obesity but remained significant (AOR, $2.38 ; P<.01)$. Post hoc tests showed that overweight/obesity and obesity may partially mediate the relationship between being American Indian and having elevated HbA1c (Sobel $z=2.42, P=$ .02 for overweight/obesity; Sobel $z=1.83, P=.07$ for obesity).

In Model 5 (Table 2), American Indians were 2.39 times as likely as non-Hispanic whites to self-report high blood glucose or diabetes $(P=.02)$, similar to the findings for elevated HbA1c. However, compared with the control variables for $\mathrm{HbAlc}$, the control variables for high blood glucose and diabetes mediated associations more strongly. In Model 6 (controls for sex, age, parent education, and parent obesity), the AOR for American Indians decreased $1.95(P=.07)$; in Model 7 (further addition of controls for neighborhood variables, urbanicity, and perceived stress), the AOR decreased to $1.83(P=.12)$, and in Model 8 (addition of overweight/obesity and obesity), it further decreased to $1.82(P=$ .14). Although none of the 3 neighborhood indicators was significantly associated with high blood glucose or diabetes, perceived stress was (AOR, 1.09; $P<.01$ ). One standard deviation increase in perceived stress was associated with a $9 \%$ increase in the likelihood of high blood glucose or diabetes, and post hoc tests confirmed perceived stress as a mediator (Sobel $z=2.23 ; P=.03$ ). Obesity did not mediate the association between being American Indian and self-reporting high blood glucose or diabetes (Sobel $z=$ $1.76, P=.08)$.

In models predicting overweight/obesity or obesity (Models 1-3) and obesity (Models 4-6) (Table 3), American Indians were more likely than non-Hispanic whites to be overweight/obese or obese, and this association was attenuated by the inclusion of covariates. In Model 6 (controls for all variables), neighborhood poverty was

The opinions expressed by authors contributing to this journal do not necessarily reflect the opinions of the U.S. Department of Health and Human Services, the Public Health Service, the Centers for Disease Control and Prevention, or the authors' affiliated institutions. 
significantly associated with obesity (AOR, 1.17, $P<.01)$. Neighborhood poverty was a partial mediator of the association between being American Indian and being obese (Sobel $z, 2.01 ; P=.05$ )

\section{Discussion}

Studies investigating social determinants or structural risk factors and the incidence of type 2 diabetes, overweight/obesity, and obesity are increasingly common $(3,10-13,19)$. Numerous studies examined social determinants or structural risk factors such as the built environment or neighborhood surroundings and their associations with such health outcomes as obesity and type 2 diabetes among racial/ethnic minority populations $(20,21)$. However, unlike other studies, our research investigated social determinants or structural risk factors that might explain the higher incidence of type 2 diabetes, overweight/obesity, and obesity among American Indians. Type 2 diabetes, overweight, and obesity are growing health concerns for American Indian adolescents and young adults. Consistent with findings from previous studies, our study provides evidence that American Indian young adults have higher rates of elevated $\mathrm{HbAlc}$ levels, self-reported type 2 diabetes or high blood glucose, and overweight/obesity or obesity than have non-Hispanic whites $(1,3)$. American Indians in our subsample also had higher rates of risk factors for poor health: they were more likely to live in neighborhoods with higher rates of poverty and housing mobility than the full sample. In addition, American Indians had higher rates of perceived stress.

Controlling for other variables, American Indian race/ethnicity was positively associated with a greater likelihood of elevated HbA1c (compared with non-Hispanic whites), and overweight/ obesity and obesity partially mediated elevated HbAlc. These results are consistent with previous research $(22,23)$. Only one of our neighborhood measures was significantly associated with $\mathrm{HbAlc}$, and it was not associated in the hypothesized direction: greater neighborhood collective efficacy predicted higher HbAlc. Previous research demonstrated an association between higher collective efficacy and decreased risk of obesity and overweight among adolescents (24). However, our Census measures were based on 1990 statistics, whereas other Wave 1 data were collected in 1994-1995. Future research should continue to explore these potential links, using more precise neighborhood indicators.

American Indians were also more likely than non-Hispanic whites to report high blood glucose or diabetes. The inclusion of control and risk factors did not mediate these associations, with the exception of stress. Perceived stress was a significant mediator of the likelihood of self-reported high blood glucose or diabetes among American Indians. Research suggests that stress may influence the onset of type 2 diabetes (25).
Consistent with other findings, neighborhood characteristics such as poverty were associated with an increased risk of high BMI in non-American Indian population groups (10,26-28). Controlling for other variables, American Indians in our study were more likely to be overweight or obese than non-Hispanic whites; high BMI among American Indians was partially mediated by neighborhood poverty. Neighborhood poverty is a risk factor for poor health; future research should examine other factors associated with neighborhood poverty, such as access to grocery stores, safety, and walkability, among American Indians $(19,20,21,26)$.

Although our study focused on the American Indian subsample, it is also interesting to compare our findings on American Indians and blacks. Without any control variables, the AOR for elevated $\mathrm{HbAlc}$ was 2.66 for American Indians and 4.86 for blacks, but in the prediction of self-reported high blood glucose or diabetes, the AORs were 2.39 for American Indians and 1.48 for blacks. Because the outcomes in these models reflect being told by a doctor, nurse, or other health care provider that one has high blood glucose or diabetes, these findings suggest that American Indians are more likely to be screened and treated for diabetes - by the Indian Health Service or others - so that they are more aware of their diabetes risk and perhaps more likely to be managing their condition. We did not measure insulin use or health insurance coverage in our study, but those issues are important for understanding differences among racial/ethnic subgroups.

This study has several limitations. First, we could not identify causal relationships between our predictors and outcomes of interest. Despite the use of longitudinal data and a robust set of control variables, our analytic strategy did not rule out the possibility of omitted variable bias. Second, our definition of the category "American Indian" combined data on participants self-reporting solely as American Indian and data on those self-identifying as American Indian in combination with one or more other groups. Future studies could investigate these American Indian subgroups separately. Third, despite the inclusion of several social determinants of health and a perceived stress indicator that might partially explain how context affects health, we did not formally test the complex pathways linking these variables to our outcomes. Future research might employ structural equation modeling or other path analyses to explore these relationships more precisely.

Despite these limitations, our study extends knowledge via several key strengths. First, the study focuses on American Indian young adults, filling a gap in the literature $(1,3,5,10-13,29)$. Much of the research examining diabetes among American Indians is dated and does not use data from large samples such as Add Health. Second, although we do not make any claims about causal relationships, the use of longitudinal data suggests that the associ-

\footnotetext{
The opinions expressed by authors contributing to this journal do not necessarily reflect the opinions of the U.S. Department of Health and Human Services, the Public Health Service, the Centers for Disease Control and Prevention, or the authors' affiliated institutions.
} 
ations between social determinants and health outcomes persist over time; this persistence points out the need for future research in this area. Third, our analytic approach of staging control variables demonstrates the extent to which certain risk factors may play a mediating role above and beyond other control variables. We hope that future research will build on this effort to estimate the effects of an improved set of social determinants among American Indian and Alaska Native populations, especially neighborhood and housing risk factors, such as safety and overcrowding.

This study emphasizes the need to further investigate the social determinants of overweight, obesity, type 2 diabetes, and elevated $\mathrm{HbA1c}$. Our research suggests that neighborhood factors and stress partially explain elevated risk for overweight, obesity, and type 2 diabetes among American Indians and that future research should include additional neighborhood factors, such as access to grocery stores selling healthy foods, proximity and safety of playgrounds or other recreational space, and adequate housing. Because neighborhood characteristics such as social capital and perceived safety are associated with lower levels of obesity in children $(10,11,20,24)$, future research should also examine these potentially protective factors at the individual, family, and community levels.

\section{Acknowledgments}

This research uses data from Add Health, a program project directed by Kathleen Mullan Harris and designed by J. Richard Udry, Peter S. Bearman, and Kathleen Mullan Harris at the University of North Carolina at Chapel Hill, and funded by grant no. P01HD31921 from the Eunice Kennedy Shriver National Institute of Child Health and Human Development, with cooperative funding from 23 other federal agencies and foundations. We especially acknowledge Ronald R. Rindfuss and Barbara Entwisle for assistance in the original design. Information on how to obtain the Add Health data files is available on the Add Health website (www.cpc.unc.edu/projects/addhealth/data). No direct support was received from grant no. P01-HD31921 for this analysis. This publication was made possible by grant no. 1P30DK092950 from the National Institute of Diabetes and Digestive and Kidney Diseases (NIDDK); its contents are solely the responsibility of the authors and do not necessarily represent the official views of NIDDK.

\section{Author Information}

Corresponding Author: Molly W. Metzger, PhD, Washington University in St Louis, George Warren Brown School of Social Work, One Brookings Dr, Campus Box 1196, St Louis, MO 63130. Telephone: 314-935-6989. Email: mmetzger22@wustl.edu.
Author Affiliation: Tennille L. Marley, Arizona State University, Tempe, Arizona.

\section{References}

1. Lau M, Lin H, Flores G. Racial/ethnic disparities in health and health care among U.S. adolescents. Health Serv Res 2012; 47(5):2031-59.

2. Ogden CL, Carroll MD, Kit BK, Flegal KM. Prevalence of obesity and trends in body mass index among US children and adolescents, 1999-2010. JAMA 2012;307(5):483-90.

3. Hamman RF, Bell RA, Dabelea D, D'Agostino RBJr, Dolan L, Imperatore $\mathrm{G}$, et al. The SEARCH for Diabetes in Youth study: rationale, findings, and future directions. Diabetes Care 2014;37(12):3336-44.

4. Frederick CB, Snellman K, Putnam RD. Increasing socioeconomic disparities in adolescent obesity. Proc Natl Acad Sci USA 2014;111(4):1338-42.

5. Acton KJ, Burrows NR, Moore K, Querec L, Geiss LS, Engelgau MM. Trends in diabetes prevalence among American Indian and Alaska Native children, adolescents, and young adults. Am J Public Health 2002;92(9):1485-90.

6. Daniels SR, Arnett DK, Eckel RH, Gidding SS, Hayman LL, Kumanyika S, et al. Overweight in children and adolescents: pathophysiology, consequences, prevention, and treatment. Circulation 2005;111(15):1999-2012.

7. Braveman P, Egerter S, Williams DR. The social determinants of health: coming of age. Annu Rev Public Health 2011; 32(1):381-98.

8. Clark ML, Utz SW. Social determinants of type 2 diabetes and health in the United States. World J Diabetes 2014; 5(3):296-304.

9. Wilkinson M, Marmot RG. Social determinants of health. New York (NY): Oxford University Press; 2006.

10. Piccolo RS, Duncan DT, Pearce N, McKinlay JB. The role of neighborhood characteristics in racial/ethnic disparities in type 2 diabetes: results from the Boston Area Community Health (BACH) survey. Soc Sci Med 2015;130:79-90.

11. Rudolph KE, Wand GS, Stuart EA, Glass TA, Marques AH, Duncko R, et al. The association between cortisol and neighborhood disadvantage in a U.S. population-based sample of adolescents. Health Place 2014;25:68-77.

12. Jacobs DE, Wilson J, Dixon SL, Smith J, Evens A. The relationship of housing and population health: a 30-year retrospective analysis. Environ Health Perspect 2009; 117(4):597-604.

13. Burton A. Does poor housing raise diabetes risk? Environ Health Perspect 2007;115(11):A534.

The opinions expressed by authors contributing to this journal do not necessarily reflect the opinions of the U.S. Department of Health and Human Services, the Public Health Service, the Centers for Disease Control and Prevention, or the authors' affiliated institutions. 
14. Harris KM. The Add Health Study: design and accomplishments; 2012. http://www.cpc.unc.edu/projects/ addhealth/data/guides/DesignPaperWIIV.pdf. Accessed March $31,2015$.

15. Chantala K. Guidelines for analyzing Add Health data; 2006. http://www.cpc.unc.edu/projects/addhealth/data/guides/wtguidelines.pdf. Accessed March 31, 2015.

16. Raudenbush SW, Sampson RJ. Ecometrics: toward a science of assessing ecological settings, with application to the Systematic Social Observation of Neighborhoods. Sociol Methodol 1999;29(1):1-41.

17. Cohen S, Kamarck T, Mermelstein R. A global measure of perceived stress. J Health Soc Behav 1983;24(4):385-96.

18. Baranowski T, Cooper DM, Harrell J, Hirst K, Kaufman FR, Goran M, et al. Presence of diabetes risk factors in a large U.S. eighth-grade cohort. Diabetes Care 2006;29(2):212-7.

19. Singh GK, Kogan MD, van Dyck PC. A multilevel analysis of state and regional disparities in childhood and adolescent obesity in the United States. J Community Health 2008; 33(2):90-102.

20.Zick CD, Smith KR, Fan JX, Brown BB, Yamada I, Kowaleski-Jones L. Running to the store? The relationship between neighborhood environments and the risk of obesity. Soc Sci Med 2009;69(10):1493-500.

21. Morland K, Wing S, Diez Roux A. The contextual effect of the local food environment on residents' diets: the Atherosclerosis Risk in Communities study. Am J Public Health 2002; 92(11):1761-7.

22. Dabelea D, Hanson RL, Bennett PH, Roumain J, Knowler WC, Pettitt DJ. Increasing prevalence of Type II diabetes in American Indian children. Diabetologia 1998;41(8):904-10.

23. Fagot-Campagna A, Saaddine JB, Flegal KM, Beckles GL. Diabetes, impaired fasting glucose, and elevated HbA1c in U.S. adolescents: the Third National Health and Nutrition Examination Survey. Diabetes Care 2001;24(5):834-7.

24. Cohen DA, Finch BK, Bower A, Sastry N. Collective efficacy and obesity: the potential influence of social factors on health. Soc Sci Med 2006;62(3):769-78.

25. Lloyd C, Smith J, Weinger K. Stress and diabetes: a review of the links. Diabetes Spectr 2005;18(2):121-7.

26. Slopen N, Non A, Williams DR, Roberts AL, Albert MA. Childhood adversity, adult neighborhood context, and cumulative biological risk for chronic diseases in adulthood. Psychosom Med 2014;76(7):481-9.

27. Fradkin C, Wallander JL, Elliott MN, Tortolero S, Cuccaro P, Schuster MA. Associations between socioeconomic status and obesity in diverse, young adolescents: variation across race/ ethnicity and gender. Health Psychol 2015;34(1):1-9.
28. Zeitler P, Fu J, Tandon N, Nadeau K, Urakami T, Barrett T, et al. Type 2 diabetes in the child and adolescent. Pediatr Diabetes 2014;15(20, Suppl 20):26-46.

29. Hutchinson RN, Shin S. Systematic review of health disparities for cardiovascular diseases and associated factors among American Indian and Alaska Native populations. PLoS ONE 2014;9(1):e80973.

\footnotetext{
The opinions expressed by authors contributing to this journal do not necessarily reflect the opinions of the U.S. Department of Health and Human Services, the Public Health Service, the Centers for Disease Control and Prevention, or the authors' affiliated institutions.
} 


\section{Tables}

Table 1. Descriptive Statistics for Full Sample and American Indian Subsample, National Longitudinal Study of Adolescent to Adult Health, 1994-2008

\begin{tabular}{|c|c|c|}
\hline Characteristic & Full Sample $(n=11,110)$ & $\begin{array}{l}\text { American Indian Subsample }(n= \\
\text { 393) }\end{array}$ \\
\hline \multicolumn{3}{|l|}{ Neighborhood (Wave 1), mean (95\% Cl) } \\
\hline Neighborhood poverty ${ }^{b}$ & $13.9(12.2-15.6)$ & $19.2(13.2-25.1)$ \\
\hline Neighborhood mobilityc & $46.5(45.0-48.0)$ & $49.3(46.5-52.0)$ \\
\hline Urban & $25.4(18.5-33.9)$ & $32.8(21.0-47.3)$ \\
\hline Suburban & $58.2(48.2-67.5)$ & $45.7(31.1-61.1)$ \\
\hline Rural & $16.4(9.5-26.9)$ & $21.4(8.3-45.0)$ \\
\hline \multicolumn{3}{|l|}{ Family (Wave 1), \% (95\% Cl) } \\
\hline \multicolumn{3}{|l|}{ Individual stress and health (Wave 4) } \\
\hline Perceived stress, mean score ${ }^{d}(95 \% \mathrm{Cl})$ & $4.8(4.7-4.9)$ & $5.6(5.2-6.0)$ \\
\hline HbA1c value, mean $(95 \% \mathrm{Cl})$ & $5.6(5.5-5.6)$ & $5.7(5.6-5.8)$ \\
\hline $\mathrm{HbA} 1 \mathrm{c} \geq 5.7, \%(95 \% \mathrm{Cl})$ & $30.6(28.4-32.8)$ & $43.8(36.4-51.5)$ \\
\hline Ever told have high blood glucose or diabetes, \% $(95 \% \mathrm{Cl})$ & $2.6(2.2-3.0)$ & $5.2(2.8-9.3)$ \\
\hline Mean body mass index, kg/m² (95\% Cl) & $29.1(28.8-29.5)$ & $30.7(29.0-32.3)$ \\
\hline Overweight or obese, \% (95\% Cl) & $66.6(64.9-68.3)$ & $76.8(69.9-82.6)$ \\
\hline Obese, \% (95\% Cl) & $37.4(35.5-39.2)$ & $42.5(33.7-51.8)$ \\
\hline
\end{tabular}

The opinions expressed by authors contributing to this journal do not necessarily reflect the opinions of the U.S. Department of Health and Human Services, the Public Health Service, the Centers for Disease Control and Prevention, or the authors' affiliated institutions. 
Table 2. Adjusted Odds Ratios From Logistic Regression Models of HbA1c and Self-Reported High Blood Glucose or Diabetes Among Young Adults ( $n=11,110)$, National Longitudinal Study of Adolescent to Adult Health, 1994-2008

\begin{tabular}{|c|c|c|c|c|c|c|c|c|}
\hline \multirow[b]{2}{*}{ Characteristic } & \multicolumn{4}{|c|}{ HbA1c (Direct Measurement) } & \multicolumn{4}{|c|}{$\begin{array}{l}\text { Diagnosis of High Blood Glucose or Diabetes } \\
\text { (Self-Reported) }\end{array}$} \\
\hline & Model 1 & Model 2 & Model 3 & Model 4 & Model 5 & Model 6 & Model 7 & Model 8 \\
\hline White [Reference] & 1.00 & 1.00 & 1.00 & 1.00 & 1.00 & 1.00 & 1.00 & 1.00 \\
\hline American Indian & $2.66^{a}$ & $2.47^{a}$ & $2.41^{a}$ & $2.38^{\mathrm{a}}$ & $2.39^{b}$ & 1.95 & 1.83 & 1.82 \\
\hline Hispanic & $2.20^{\mathrm{a}}$ & $2.07^{a}$ & $2.15^{\mathrm{a}}$ & $2.00^{a}$ & 1.57 & 1.25 & 1.26 & 1.13 \\
\hline Asian & $2.06^{a}$ & $2.30^{\mathrm{a}}$ & $2.42^{\mathrm{a}}$ & $2.69^{a}$ & 0.43 & 0.50 & 0.50 & 0.58 \\
\hline Other race/ethnicity & $2.25^{\mathrm{a}}$ & $2.33^{a}$ & $2.38^{\mathrm{a}}$ & $2.44^{\mathrm{a}}$ & 0.71 & 0.66 & 0.65 & 0.62 \\
\hline Male & - & $1.78^{\mathrm{a}}$ & $1.78^{\mathrm{a}}$ & $1.80^{\mathrm{a}}$ & - & 0.74 & 0.78 & 0.80 \\
\hline High school diploma & - & $0.82^{b}$ & $0.83^{b}$ & $0.82^{b}$ & - & 0.60 & 0.64 & 0.63 \\
\hline Some college & - & $0.72^{\mathrm{a}}$ & $0.75^{\mathrm{a}}$ & $0.76^{\mathrm{a}}$ & - & $0.56^{b}$ & 0.61 & 0.63 \\
\hline College diploma & - & $0.63^{a}$ & $0.66^{b}$ & $0.70^{\mathrm{a}}$ & - & $0.40^{\mathrm{a}}$ & $0.45^{\mathrm{a}}$ & $0.49^{\circ}$ \\
\hline >College diploma & - & $0.61^{a}$ & $0.65^{a}$ & $0.71^{b}$ & - & $0.28^{a}$ & $0.32^{b}$ & 0.38 \\
\hline Parent is obese & - & $1.52^{\mathrm{a}}$ & $1.52^{\mathrm{a}}$ & $1.25^{\mathrm{a}}$ & - & $1.72^{\mathrm{a}}$ & $1.70^{\mathrm{a}}$ & 1.36 \\
\hline \multicolumn{9}{|c|}{ Neighborhood characteristics $^{c}$} \\
\hline $\begin{array}{l}\text { Neighborhood collective } \\
\text { efficacy }^{d}\end{array}$ & - & - & $1.07^{b}$ & $1.07^{b}$ & - & - & 1.02 & 1.01 \\
\hline Perceived stress ${ }^{c, g}$ & - & - & 1.01 & 1.01 & - & - & $1.09^{a}$ & $1.09^{\circ}$ \\
\hline
\end{tabular}

Abbreviation: -, not applicable; HbA1c, glycated hemoglobin.

a $P<.01$.

b $P<.05$.

${ }^{c}$ Calculated as $z$ scores, normed such that the mean equals zero and standard deviation equals 1 . Coefficients can be interpreted as the adjusted odds ratio associated with a 1 standard deviation increase in the predictor.

${ }^{d}$ A measure of social cohesion scored on a scale of 0 to 3 , with a higher score indicating better neighborhood efficacy.

e Percentage of people living below the official poverty threshold, based on 1990 Census block group data.

${ }^{f}$ Measured as the percentage of occupied housing units into which people moved during the previous 5 years, based on 1990 Census block group data.

$g$ Measure of stress based on the Cohen Perceived Stress Scale (PSS) $(17,18)$. Scored on a scale of 0 to 16 , with higher scores indicating greater stress.

(continued on next page)

The opinions expressed by authors contributing to this journal do not necessarily reflect the opinions of the U.S. Department of Health and Human Services, the Public Health Service, the Centers for Disease Control and Prevention, or the authors' affiliated institutions. 
(continued)

Table 2. Adjusted Odds Ratios From Logistic Regression Models of HbA1c and Self-Reported High Blood Glucose or Diabetes Among Young Adults $(n=11,110)$, National Longitudinal Study of Adolescent to Adult Health, 1994-2008

\begin{tabular}{|l|r|r|r|r|r|r|r|}
\hline \multirow{2}{*}{ Characteristic } & \multicolumn{4}{|c|}{ HbA1c (Direct Measurement) } & \multicolumn{3}{c|}{ Diagnosis of High Blood Glucose or Diabetes } \\
(Self-Reported)
\end{tabular}

Abbreviation: -, not applicable; HbA1c, glycated hemoglobin.

a $P<.01$.

b $P<.05$.

${ }^{\mathrm{c}}$ Calculated as $z$ scores, normed such that the mean equals zero and standard deviation equals 1 . Coefficients can be interpreted as the adjusted odds ratio associated with a 1 standard deviation increase in the predictor.

${ }^{d}$ A measure of social cohesion scored on a scale of 0 to 3 , with a higher score indicating better neighborhood efficacy.

e Percentage of people living below the official poverty threshold, based on 1990 Census block group data.

${ }^{f}$ Measured as the percentage of occupied housing units into which people moved during the previous 5 years, based on 1990 Census block group data.

$g$ Measure of stress based on the Cohen Perceived Stress Scale (PSS) $(17,18)$. Scored on a scale of 0 to 16 , with higher scores indicating greater stress.

The opinions expressed by authors contributing to this journal do not necessarily reflect the opinions of the U.S. Department of Health and Human Services, the Public Health Service, the Centers for Disease Control and Prevention, or the authors' affiliated institutions. 
Table 3. Adjusted Odds Ratios From Logistic Regression Models of Overweight and Obesity, National Longitudinal Study of Adolescent to Adult Health $(n=11,110), 1994-2008$

\begin{tabular}{|c|c|c|c|c|c|c|}
\hline Characteristic & \multicolumn{3}{|c|}{ Overweight/Obesity } & \multicolumn{3}{|c|}{ Obesity } \\
\hline \multicolumn{7}{|l|}{ Race/ethnicity } \\
\hline White [Reference] & 1.00 & 1.00 & 1.00 & 1.00 & 1.00 & 1.00 \\
\hline American Indian & $1.87^{\mathrm{a}}$ & $1.62^{b}$ & $1.65^{\mathrm{a}}$ & $1.38^{b}$ & 1.15 & 1.09 \\
\hline Asian & 0.67 & 0.81 & 0.84 & $0.51^{a}$ & $0.64^{b}$ & 0.67 \\
\hline Other race/ethnicity & 0.96 & 0.94 & 0.96 & $1.11^{\mathrm{a}}$ & 1.07 & 1.08 \\
\hline Male & - & $1.48^{a}$ & $1.46^{\mathrm{a}}$ & - & 0.93 & 0.93 \\
\hline Age & - & $1.06^{a}$ & $1.06^{a}$ & - & $1.05^{a}$ & $1.05^{a}$ \\
\hline Some college & - & 0.85 & 0.86 & - & 0.84 & 0.90 \\
\hline College diploma & - & $0.68^{a}$ & $0.69^{a}$ & - & $0.67^{a}$ & $0.74^{a}$ \\
\hline >College diploma & - & $0.58^{a}$ & $0.59^{a}$ & - & $0.52^{a}$ & $0.59^{a}$ \\
\hline Parent is obese & - & $2.43^{a}$ & $2.44^{a}$ & - & $2.47^{\mathrm{a}}$ & $2.48^{a}$ \\
\hline \multicolumn{7}{|l|}{ Neighborhood characteristics ${ }^{c}$} \\
\hline Neighborhood collective efficacy ${ }^{d}$ & - & - & 1.02 & - & - & 1.02 \\
\hline Neighborhood povertye & - & - & 1.06 & - & - & $1.17^{a}$ \\
\hline Neighborhood mobility ${ }^{f}$ & - & - & 0.99 & - & - & 0.96 \\
\hline Constant & 1.78 & 0.63 & 0.70 & 0.54 & 0.25 & 0.24 \\
\hline
\end{tabular}

a $P<.01$.

${ }^{\mathrm{b}} P<.05$.

${ }^{\mathrm{c}}$ Calculated as $z$ scores, normed such that the mean equals zero and standard deviation equals 1 . Coefficients can be interpreted as the adjusted odds ratio associated with a 1 standard deviation increase in the predictor.

${ }^{d}$ A measure of social cohesion scored on a scale of 0 to 3 , with a higher score indicating better neighborhood efficacy.

e Percentage of people living below the official poverty threshold, based on 1990 Census block group data.

${ }^{f}$ Measured as the percentage of occupied housing units into which people moved during the previous 5 years, based on 1990 Census block group data.

$g$ Measure of stress based on the Cohen Perceived Stress Scale (PSS) $(17,18)$. Scored on a scale of 0 to 16 , with higher scores indicating greater stress.

The opinions expressed by authors contributing to this journal do not necessarily reflect the opinions of the U.S. Department of Health and Human Services, the Public Health Service, the Centers for Disease Control and Prevention, or the authors' affiliated institutions. 